FORMATION Formation emploi

Revue française de sciences sociales

133 | Janvier-Mars 2016

Heurs et malheurs de l'apprentissage en Suisse

\title{
Introduction : les faux-semblants de l'apprentissage en Suisse
}

Nadia Lamamra et Gilles Moreau

\section{(2) OpenEdition}

1 Journals

Édition électronique

URL : http://journals.openedition.org/formationemploi/4636

DOI : 10.4000/formationemploi.4636

ISSN : 2107-0946

Éditeur

La Documentation française

Édition imprimée

Date de publication : 20 avril 2016

Pagination : 7-16

ISSN : 0759-6340

Référence électronique

Nadia Lamamra et Gilles Moreau, "Introduction : les faux-semblants de l'apprentissage en Suisse », Formation emploi [En ligne], 133 | Janvier-Mars 2016, mis en ligne le 20 avril 2016, consulté le 10 décembre 2020. URL : http://journals.openedition.org/formationemploi/4636 ; DOI : https://doi.org/ 10.4000/formationemploi.4636

(c) Tous droits réservés 


\title{
Introduction
}

\section{Les faux-semblants de l'apprentissage en Suisse}

\author{
NADIA LAMAMRA \\ Sociologue, Institut fédéral des hautes études en formation professionnelle, EHB IFFP IUFFP, \\ Lausanne \\ Gilles Moreau \\ Sociologue, Groupe de recherches et d'études sociologiques du Centre Ouest, GRESCO, \\ université de Poitiers
}

Ces dernières années, la formation professionnelle, et en particulier l'apprentissage, est au centre de l'attention politique en France. Les différents gouvernements, aux prises avec la crise économique et un chômage juvénile récurrent, y voient une solution aux difficultés d'insertion professionnelle des jeunes, au point qu'un objectif de 500000 apprentis est régulièrement affiché, sans succès jusque-là. En la matière, pour la France comme pour les pays membres de l'Union européenne, le pays de référence reste encore fréquemment l'Allemagne ${ }^{1}$.

Pour autant, le modèle de formation professionnelle helvétique, souvent associé à la rhétorique du "miracle suisse " ${ }^{2}$, ne cesse de gagner en légitimité hors de ses frontières : ainsi, le Président François Hollande, lors de son voyage en Suisse en avril 2015, a retenu de ses visites d'entreprises une volonté de développer l'alternance dans les entreprises françaises ${ }^{3}$. De même, à l'issue d'un voyage aux Etats-Unis, en juillet 2015, le ministre suisse de l'Economie (dont dépend la formation professionnelle), Johann Schneider-Ammann, a finalisé un accord sur la formation entre les deux Etats : le système dual helvétique séduit même outre-Atlantique ${ }^{4}$.

1. En témoigne un récent rapport produit en 2015 (Rapport d'information $\mathrm{n}^{\circ} 719$, enregistré à la Présidence du Sénat le 30 septembre 2015).

2. Rappelons, à ce titre, l'émission «Un oeil sur la planète ", diffusée par France 2, le 16 décembre 2013, portant sur "Le miracle suisse " ou encore l'ouvrage de François Garçon, L'autre miracle suisse, paru aux Presses polytechniques et universitaires romandes, en 2014.

3. François Nordmann, "Bilan d'une visite réussie ", Le Temps, 20 avril 2015, consulté le 11.01 .16 sur http://letemps.ch/opinions/2015/04/20francois-hollande-bilan-une-visite-reussie. Isabelle Biolley et Renaud Bournoud, A l'EPFL, François Hollande salue le «miracle suisse». 24 Heures, 14 avril 2015, consulté le 11.01.16 sur : http://www.24heures.ch/vaud-regions/lausanne-region/epfl-francois-hollande-salue-miraclesuiss/story/20819348.

4. Coralie Claude, "La Suisse va devenir "maitre d'apprentissage" des Etats-Unis », RTS Info (Radio télévision suisse), Forum, le 9 juillet 2015, consulté le 11.01.16 sur http://www.rts.ch/info/economie/6931919la-suisse-va-devenir-maitre-d-apprentissage-des-etats-unis.html. La rédaction, " Les Etats-Unis comptent sur la Suisse pour faire de l'apprentissage une priorité ", Le Temps, le 9 juillet 2015, consulté le 11.01.16 sur http://www.letemps.ch/economie/2015/07/09/etats-unis-comptent-suisse-faire-apprentissage-une-priorite. 
Penser le système de formation suisse comme un modèle n'est pas uniquement le produit de l'intérêt qu'y portent les pays étrangers ; c'est aussi le fruit d'une stratégie des autorités helvétiques dans leur politique de coopération internationale en matière de formation professionnelle (Confédération suisse, 2014).

Ainsi, nombre de délégations étrangères viennent voir ce "modèle de réussite " qui leur est souvent présenté ainsi : une part importante des jeunes se formant dans la voie professionnelle en système dual, un faible taux de chômage juvénile et des possibilités de formation supérieure liées à la voie professionnelle, y compris en formation continue. La formation professionnelle suisse serait-elle en passe de devenir, avec les banques et l'horlogerie, un des principaux produits d'exportation du pays?

La plus grande visibilité du système suisse, ses changements et développements récents se sont accompagnés d'un intérêt croissant de la recherche pour cette voie de formation. Longtemps délaissée, en particulier par les sciences sociales (Imdorf et alii, 2010), la formation professionnelle des jeunes suisses fait désormais l'objet d'une attention grandissante. Preuve en est la mise en place de programmes de recherches (Leadinghouses) financés par le Secrétariat d'Etat à la formation, à la recherche et à l'innovation (SEFRI), ainsi que le développement de projets menés par différentes institutions de recherche publiques et privées $^{5}$.

Ce numéro de Formation Emploi s'inscrit dans cette dynamique : dépasser l'illusio du " miracle " helvétique pour dire ce qu'est l'apprentissage en suisse et non ce qui en est dit, en diffusant des recherches qui visent à mieux cerner et analyser la réalité de la formation professionnelle suisse, et en priorité la formation initiale ${ }^{6}$. A partir d'enquêtes empiriques, ce numéro vise à dévoiler les mécanismes à l'œuvre derrière le " miracle helvétique ", à interroger les impensés du système, ses angles morts, bref à nuancer nombre d'idées reçues associées au modèle suisse et à réaffirmer ainsi le primat de la raison critique sur l’angélisme politique.

5. Econcept AG \& Gonon P. (2014), Evaluation de la recherche sur la formation professionnelle encouragée par le SEFRI. Résumé du rapport final et recommandations, Berne : Secrétariat d'État à la formation, à la recherche et à l'innovation (SEFRI). Même si elle n'a pas été menée prioritairement en vue de documenter les parcours en formation professionnelle, l'étude TREE (Transition école-emploi) doit être signalée ici. Il s'agit de la première étude longitudinale au niveau national sur la transition des jeunes de l'école à l'emploi. Le premier échantillon (TREE1) comprend plus de 6000 jeunes qui ont participé à l'enquête PISA (Programme for International Student Assessment) en 2000 et qui ont achevé leur formation en école obligatoire la même année. Cet échantillon est représentatif tant sur le plan national qu'au niveau des régions linguistiques. Une deuxième cohorte (TREE2) sera suivie à partir de 2016.

6. Ce numéro porte principalement sur la formation professionnelle initiale. Les apprenti-e-s sont des jeunes se formant majoritairement en alternance ou, de façon minoritaire, en écoles plein-temps (type lycées professionnels). En Suisse, les stages suivis au niveau tertiaire (supérieur) ne relèvent pas du système dual. A noter que Formation Emploi a déjà consacré un numéro à la formation en Suisse $\left(\mathrm{n}^{\circ} 100,2007\right)$, mais il s’agissait alors principalement de la formation continue. 
Se dévoilent ainsi un système de formation puissant mais inégalitaire, une articulation étroite entre l'apprentissage et le marché du travail, mais également une implication très variable des entreprises dans le dispositif de formation, ainsi que des défis nouveaux pour l'avenir.

\section{Un système de formation incontournable, mais profondément inégalitaire}

En Suisse, près des deux-tiers des jeunes s'orientent ou sont orienté-e-s vers la voie professionnelle au terme de leur formation secondaire, soit vers 15 ans.

$\mathrm{Au}$ sein de cette population, près de $80 \%$ optent pour le système dit dual, qui alterne formation théorique en école professionnelle (l'équivalent des Centres de formation d'apprentis - CFA - en France) et formation pratique en entreprise. Les $20 \%$ restant sont accueillis en écoles dites à " plein-temps ", où la formation, tant pratique que théorique, se déroule en établissements scolaires (l'équivalent des lycées professionnels en France).

Ces deux parcours de formation ne sont pas strictement équivalents dans la mesure où la forme scolaire y est plus ou moins prégnante, même si, comme le montre l'article que consacre, dans ce numéro, Najate Zouggari aux apprenti-e-s ébénistes, les frontières entre logiques scolastiques et logiques d'apprentissage in situ demeurent poreuses. La Suisse n'adopte d'ailleurs pas une position strictement homogène face à la formation : l'apprentissage dual domine dans la partie alémanique et la formation en école est plus fréquente en Romandie ; de façon générale, le plébiscite de la formation professionnelle est plus marqué dans la partie germanophone du pays et la voie académique (ou généraliste) davantage privilégiée en Suisse latine (francophone et italophone).

Vue de France, cette orientation massive des jeunes suisses vers la formation professionnelle initiale est étonnante, voire inimaginable. Mais en Suisse, elle est en trompe-l'œil : en effet, les chiffres laissent entendre que deux jeunes sur trois, tous milieux sociaux confondus, optent pour la voie professionnelle; se renforce ainsi l'illusion d'une Suisse sans stratification sociale, sans antagonismes et sans classes (Levy, Joye, Guye \& Kaufmann, 1997).

Il est vrai, comme le rappelle Lorenzo Bonoli dans son article, qu'historiquement, la Suisse a pensé la formation professionnelle non seulement dans le but de former une main-d'œuvre qualifiée, mais aussi dans celui de contenir les antagonismes et maintenir la paix sociale.

Or, derrière cette volonté se cache une réelle reproduction sociale. Ainsi, la voie professionnelle demeure le destin probable des enfants de milieux ouvriers et employés, ainsi que celui des enfants issu-e-s de familles de petit-e-s indépendant-e-s. 
Si la fracture est moins nette qu'en France, où la voie professionnelle et l'apprentissage en entreprise sont, aux niveaux V et IV, "réservés » à la jeunesse populaire (Moreau, 2003 ; Palheta 2012), la frontière demeure marquée en Suisse : les jeunes issu-e-s des classes supérieures de la société se concentrent dans les filières académiques (gymnase - équivalent du lycée général - et université) et sont sous-représenté-e-s dans le système dual et les écoles professionnelles à «temps plein ».

La difficulté à obtenir des données statistiques nationales pour mesurer ce phénomène constitue un obstacle de taille pour la recherche, mais certaines données cantonales, comme celles que présentent Rami Mouad et François Rastoldo dans ce numéro, rendent compte de ces inégalités sociales. Ces dernières reposent, institutionnellement, sur une sélection scolaire très précoce des jeunes, en Suisse. En effet, le choix ou l'assignation à l'une des filières du secondaire est déterminant pour la poursuite en voie professionnelle ou en voie générale. . Or, cette orientation s'opère vers l'âge de 12 ans.

Au final, orientation précoce et forte sélectivité viennent invalider les éventuels effets positifs de la massification scolaire qu'a connue la Suisse depuis une vingtaine d'années, maintenant ainsi l'ordre social malgré les récentes réformes de la formation et du système éducatif. L'article que consacre Julie Falcon à cette question l'illustre bien.

\section{Un lien étroit avec le marché du travail}

La particularité du modèle suisse, et notamment de son système dual, est le lien étroit entre formation et marché du travail, par l'entremise de trois partenaires : la Confédération (la formation professionnelle est le seul système de formation en Suisse qui soit réglementé au niveau fédéral), les Cantons (responsables de la mise en œuvre, de la relation avec les entreprises formatrices et des écoles professionnelles) et enfin, les Organisations du monde du travail (associations professionnelles, organisations patronales et syndicales). Ces trois

7. La scolarité obligatoire étant régie au niveau cantonal, de fortes disparités existent. Cependant, depuis 2009, un concordat intercantonal (Harmos) est entré en vigueur. Il a uniformisé, en Suisse, la durée et l'organisation de l'enseignement obligatoire, qui se structure comme suit : onze années de formation, soit huit années de primaire (organisées en deux cycles, qui correspondent aux standards de l'OCDE - Organisation de coopération et de développement économiques - ISCED 0 et ISCED1 - International Standard Classification Of Education) et trois années de secondaire (ISCED 2). La formation secondaire (aussi appelée secondaire I) diffère selon les cantons. Elle est organisée en deux ou trois filières selon les niveaux d'exigence (élevé, moyen, faible). Ces filières définissent, pour une grande part, les orientations futures. Un point commun est à souligner : quel que soit le nombre de filières, dans chaque canton, l'une prépare toujours à la voie académique conduisant directement au gymnase (lycée), puis à l'université. Avec la réforme Harmos, l'orientation dans des filières a été retardée. Elle intervient vers l'âge de 12-13 ans, alors que précédemment elle s’opérait déjà vers 11 ans. 
partenaires élaborent les ordonnances (mise en œuvre de la Loi), rédigent les plans de formation et s'occupent des différentes révisions.

Au niveau cantonal, les associations professionnelles et patronales, et souvent directement les entreprises, sont fortement sollicitées, tout comme les maître-s-ses d'apprentissage qui, en Suisse, sont obligé-e-s de suivre une formation pédagogique ${ }^{8}$.

Elles sont impliquées également dans la mise en place des cours interentreprises, nouveau pilier du système, que d'aucun-e-s qualifient désormais de trial (les cours interentreprises constituant le troisième lieu de formation, en plus de l'école et de l'entreprise).

Cette organisation, dans laquelle les entreprises pourvoyeuses de places d'apprentissage sont centrales, explique le lien étroit entre formation et marché du travail, et la prise en compte relative - l'apprentissage permet aussi de disposer d'une main-d'œuvre pas chère des réquisits de l'économie. Elle permet enfin d'être plus réactif face aux changements qui interviennent sur le marché du travail.

Mais, envers du miroir, cette organisation suit les mêmes logiques que ce dernier, en termes de ségrégations, d'inégalités et de discriminations, ainsi que le soulignent les articles d'Isabelle Zinn dans les métiers de la boucherie et de Barbara Duc, à travers une analyse originale de la relation entre l'apprenti et son maître d'apprentissage.

Ce système génère un véritable marché des places d'apprentissage, sur le modèle du marché du travail. Lors de la recherche d'un contrat d'apprentissage, les jeunes doivent se positionner comme le ferait un demandeur d'emploi, dans un marché ségrégué où la sélection, et par conséquent la concurrence, est sévère. Guillaume Ruiz et Gaële Goastellec consacrent un article à cette question en montrant néanmoins que les normes formelles (adéquation aux réquisits scolaires, conformité aux normes de comportement, etc.) ne rendent pas totalement compte des processus de recrutement, ceux-ci se fondant également sur des éléments plus subjectifs.

Si à certaines périodes (fin des années 90 - début des années 2000), le marché des places d'apprentissage a connu, en Suisse, un épisode de pénurie, le décalage qualitatif entre l'offre et la demande demeure un phénomène récurrent. Ainsi, certains secteurs peinent à recruter des jeunes, peu attiré-e-s par des activités socialement dévalorisées et/ou des conditions de travail difficiles (métiers du bâtiment, de la restauration et de l'hôtellerie, boucherie, notamment), pendant que d'autres sont saturés (emplois de bureaux, métiers de l'informatique par exemple) et peuvent élever leur niveau de sélection.

8. Pour qu'une entreprise obtienne, de la part du canton, l'autorisation de former, elle doit disposer d'une ou de plusieurs personnes qualifiées. Pour obtenir cette qualification, les futur-e-s maîtres-ses d'apprentissage suivent une formation de $40 \mathrm{~h}$ minimum. Cette formation, obligatoire, est organisée par les cantons ou les associations professionnelles. Chaque branche définit les conditions minimales pour devenir formateur ou formatrice (généralement le nombre d'années d'expérience dans le métier) et les conditions d'exercice (nombre d'apprenti-e-s dont une personne peut s'occuper). 
Les secteurs délaissés se voient contraints à rendre leurs métiers attractifs grâce à des campagnes de promotion, en se réorganisant, notamment par la création de nouvelles spécialités de formation. Le but est d'attirer un public plus large, y compris des jeunes femmes, dans les "bastions masculins ". Sous cet aspect d'ailleurs, il n'y a pas de "miracle suisse " : la formation professionnelle s'y caractérise, comme en France, par une forte ségrégation de sexe et une quasi-absence de mixité (Lamamra, 2016). Au final, les efforts de revalorisation des métiers et de création de nouvelles spécialités accessibles aux femmes ne modifient pas fondamentalement la sexuation des filières de formation; au mieux, ils conduisent à une reconfiguration de la division sexuelle du travail, un aspect que souligne parfaitement la contribution d'Isabelle Zinn.

\section{Une implication variable 3 des entreprises}

La forte implication des entreprises dans le système dual suisse ne doit pas faire oublier que toutes ne participent pas activement à la formation professionnelle, loin de là. Seules environ un tiers d'entre elles forment des apprenti-e-s' ${ }^{9}$, ce qui nuance grandement l'image d'entreprises suisses marchant comme un seul homme derrière son système de formation.

Nombre d'entreprises ne souhaitent pas "prendre ce risque » ou encore relèvent de secteurs où il n'existe aucune tradition de formation et/ou dans lesquels le mode d'accès ne passe pas par l'apprentissage, mais par la voie académique (universités notamment).

Si un certain nombre de grandes entreprises " jouent le jeu " de la formation, mettant en avant des centres de formation de qualité, la majorité des entreprises formatrices sont en fait des PME, avec une prédominance de micro-entreprises (moins de dix équivalents temps plein - ETP) et de petites entreprises (de 10 à 49 ETP). Ces dernières cherchent d'ailleurs à réduire au maximum les risques et pratiquent donc un recrutement très sélectif, inégalitaire et discriminant (Imdorf, 2007).

9. Le taux d'entreprises formatrices dépend du mode de calcul : prise en compte ou non des micro-entreprises, exclusion de certains types d'entreprises, etc. Dans l'étude sur les coûts/bénéfices de la formation des apprenti-e-s, Strupler et Wolter (2012) ont ainsi exclu de leur échantillon les entreprises ultra spécialisées qui expliquaient que ce haut degré de spécialisation rendait la formation d'apprenti-e-s impossible. Ces auteurs parviennent alors à un taux de $42 \%$. A partir du recensement fédéral des entreprises, Müller et Schweri (2012) obtiennent eux un taux de 18,4\%. Depuis 2011, la nouvelle Statistique structurelle des entreprises (STATENT) a remplacé le recensement des entreprises. Toutes les structures, y compris les plus petites, sont intégrées et le pourcentage d'entreprises formatrices est encore inférieur à celui indiqué par Müller et Schweri. Ainsi, à nombre d'entreprises constant, le rapport du nombre d'entreprises formatrices, exprimé sur la population des entreprises actives, est de 9,9\%, soit bien supérieur à ce qu'il a pu être en 2008 avec les chifffres du dernier recensement des entreprises. 
Face à la réticence d'une large partie des entreprises à prendre des apprenti-e-s, des innovations ont vu le jour ces dernières années. Ainsi, des " réseaux d'entreprises formatrices » permettent à des employeurs de s'organiser, se partageant les charges de recrutement, l'administration et le suivi des apprenti-e-s tout au long de leur formation professionnelle. Christian Imdorf, Sandra Da Rin et Regula Leemann consacrent un article à ces réseaux, inconnus en France, en révèlent l'intérêt, notamment la variété d'expériences que peuvent ainsi vivre les apprenti-e-s, mais aussi les tensions que génère cette innovation, dans la mesure où les entreprises ainsi associées ne sont pas toujours mûes par des intérêts communs.

\section{Des défis pour l'avenir : des transitions difficiles et différenciées selon le niveau de formation}

Vanté pour sa préparation adaptée au marché du travail, le système dual suisse est néanmoins confronté à de nouveaux défis. En effet, l'un des atouts majeurs de l'apprentissage en entreprise était de permettre une transition douce et aménagée de l'école au marché du travail (Cohen-Scali, 2000), l'élève devenu-e apprenti-e entrant peu à peu dans le monde et les logiques du travail.

Or, depuis une quinzaine d'années, ce n'est plus le cas : le processus de transition pour accéder à l'apprentissage puis à l'emploi s'allonge et connaît une complexification croissante (Bergman \& alii, 2011). Dès lors, le phénomène de la transition école-travail devient objet de débats politiques et de recherche.

La situation a en effet beaucoup évolué : le passage direct en apprentissage au sortir du secondaire obligatoire est plus rare, les aspirant-e-s apprenti-e-s connaissant à présent un temps de latence relativement long (l'âge moyen de sortie du secondaire obligatoire est de 15 ans $^{10}$, alors que celui d'entrée en apprentissage est de 16,5-17,5 ans ${ }^{11}$ ).

Ce phénomène d'allongement du premier seuil de transition, appelé " Transition I ", a conduit les pouvoirs publics à mettre en place diverses mesures (allongement de la scolarité, institutions d'aides à l'insertion ou encore espaces d'élaboration du projet professionnel). Loin du passage en pente douce vers le monde du travail, la "Transition I "

10. Département de la formation et de la jeunesse (DFJ), «L'école obligatoire. Le cursus scolaire », Canton de Vaud 2015, consulté le 11 janvier 2016 sur : http://www.vd.ch/themes/formation/scolarite-obligatoire/ cursus-scolaire/.

11. Office Fédéral de la Statistique, "Âge moyen des entrants au secondaire II, 1990-2013 ", consulté le 11 janvier 2016 sur : http://www.bfs.admin.ch/bfs/portal/fr/index/themen/15/17/blank/01.indicator.404102.4034.html. Les personnes en voie CFC entrent en moyenne à l'âge de 16,5 ans, les personnes se dirigeant vers une filière AFP commencent en moyenne une année plus tard, à l'âge de 17,5 ans. De plus, des écarts importants sont à noter, certains cantons connaissant des entrées au secondaire II professionnel plus tardives. 
confronte aujourd'hui certain-e-s jeunes au chômage, à la précarité, mais aussi aux discriminations et aux inégalités face à un marché de l'apprentissage qui leur est de moins en moins accessible.

A ce premier seuil délicat (Transition I), s'ajoutent parfois des parcours de formation chaotiques, marqués par des réorientations ou des ruptures de contrat, nombreuses en Suisse comme en France (Lamamra \& Masdonati, 2009). Mais le second passage, de la formation professionnelle à l'emploi, dit de "Transition II ", est lui aussi souvent difficile à négocier. En Suisse, en raison d'un taux de chômage des jeunes (comme des adultes) très bas ${ }^{12}$, la question de l'insertion professionnelle est rarement posée dans la sphère publique.

Pourtant, les travaux portant sur la « Transition II » questionnent la qualité de cette insertion. Il en ressort d'importantes disparités. En effet, selon le niveau de formation et le type de filière suivie (en système dual ou en école), les chances d'obtenir un emploi et de s'y maintenir varient. Rami Mouad et François Rastoldo le montrent bien à partir d'une étude détaillée dans les cantons de Genève et de Vaud, tout comme l'article d'Helen Buchs et Barbara Müller, basé sur l'enquête de suivi de cohorte TREE ${ }^{13}$. Dans les deux cas, plus le niveau de formation sera élevé ${ }^{14}$, meilleures seront les chances d'insertion. La formation pratique ne fait pas tout : le système des diplômes professionnels - et sa hiérarchisation récente - joue aussi dans le destin social des jeunes, en Suisse... comme en France (Millet \& Moreau, 2011).

Longtemps réputée au travers d'un diplôme de référence quasi monopolistique, le Certificat fédéral de capacité (CFC), la formation professionnelle suisse a entamé, à la fin du XX siècle, une mue importante, nourrie, entre autres, par les comparaisons internationales des systèmes éducatifs (classements PISA - Programme international pour le suivi des acquis des élèves - enquêtes de l'OCDE, etc.), qui engendrent de véritables mises en compétition des systèmes de formation.

Tout en cherchant à exporter son modèle de formation professionnelle initiale, la Suisse a dû se confronter à l'élévation générale des niveaux de formation en Europe. La formation professionnelle connaît, dès lors, une élévation des niveaux d'exigences dans toutes ses filières, qualifiée par certain-e-s d'intellectualisation (Moser, 2004).

Ainsi, dès la fin des années 1990, une réforme du système a été amorcée. Elle s’est traduite, d'une part, par la création d'un diplôme supérieur et complémentaire au CFC, la Maturité professionnelle (Gonon, 2013) ; d'autre part, par le développement des Hautes écoles spécialisées (HES), permettant une formation professionnelle supérieure d'un genre nouveau.

12. Au troisième trimestre 2015, en Suisse, le taux de chômage était de 4,9\%. Le taux de chômage des jeunes (15 à 24 ans) était, pour la même période, de 10,6 \% (OFS, 2015).

13. Cf. note 6 .

14. Il existe trois diplômes accessibles par apprentissage dual en Suisse : le CFC, diplôme historique, et deux nouveaux diplômes créés à la fin du XXe siècle : l'AFP et la Maturité professionnelle, diplômes situés respectivement en amont et en aval du CFC. 
Plus récemment, la mise en œuvre d'un nouveau diplôme en deçà du CFC, l'Attestation fédérale de formation professionnelle (AFP), a permis de fixer des objectifs politiques ambitieux : atteindre $95 \%$ de diplômé-e-s du secondaire II. Donnant accès à une formation professionnelle moins exigeante à des jeunes moins bien doté-e-s scolairement, ce nouveau diplôme permet, théoriquement, d'offrir une filière complète dans la voie professionnelle : AFP, CFC, Maturité professionnelle. Cependant, ces changements contribuent à déstabiliser le CFC, qui n'est désormais plus le premier diplôme d'accès au marché de l'emploi, et dont la finalité est devenue plus floue ; il est en effet à la fois diplôme d'insertion et diplôme propédeutique permettant de poursuivre vers la Maturité professionnelle et les HES, comme en témoignent, dans ce numéro, Nadia Lamamra et Gilles Moreau. D’une certaine manière, le CFC est confronté à ce qu'ont pu connaître, en France, le CAP (certificat d'aptitude professionnelle) et le BEP (brevet d'études professionnelles) avec la création du baccalauréat professionnel (Brucy, Maillard, Moreau, 2013).

Si ces changements semblent offrir de nouvelles perspectives de formation, ils réaffirment avant tout une hiérarchisation et une division sociale du travail accrues. La formation professionnelle suisse se retrouve désormais face à un paradoxe : vanter la formation supérieure sans pour autant dévaloriser la formation initiale de niveau secondaire.

Pour aborder ces enjeux, ce numéro de Formation Emploi fait la part belle à des recherches de terrain menées tant en Suisse romande qu'alémanique. Les auteur-e-s sont issu-e-s de différentes disciplines, histoire, sociologie, sciences de l'éducation, ethnographie, et optent pour des approches diverses : études quantitatives, analyse secondaire de données statistiques, entretiens, observations, etc.

Leurs articles révèlent, au-delà de l'image d'Epinal, comment et à quel prix s'est construit le modèle de formation professionnelle suisse, les tensions, contradictions, inégalités et paradoxes qui l'habitent, mais également les capacités d'innovation qui le caractérisent.

\section{Bibliographie}

Bergman M. M., Hupka-Brunner S., Keller A., Meyer T. \& Stalder B. E. (Eds.), (2011). Youth transitions in Switzerland. Results from the TREE Panel Study, Berne, Seismo.

Brucy G., Maillard F., Moreau G. (2013), Le CAP, un diplôme du peuple. 1911-2011, Rennes, Presses universitaires de Rennes.

Cohen-Scali V. (2000), Alternance et identité professionnelle, Paris, Presses universitaires de France.

Confédération suisse (2014), Coopération internationale en matière de formation professionnelle (CIFP). Concrétisation de la stratégie internationale FRI de la Suisse dans le domaine de la formation professionnelle, Berne : Département de l'économie, de la formation et 
de la recherche DEFR / Secrétariat d'État à la formation, à la recherche et à l'innovation (SEFRI).

Formation Emploi (2007), "De la formation professionnelle en Suisse ", Dossier, n ${ }^{\circ} 100$, octobre-décembre, Paris, La Documentation française.

Gonon Ph. (2013), "Federal Vocational Baccalaureate: The Swiss Way of Hybridity", in Deissinger Th., Aff J., Fuller A. \& Helms Jorgensen Ch. (Eds.): Hybrid Qualifications - Structures and Problems in the Context of European VET Policy, Berne, Peter Lang, pp. 181-196.

Imdorf C., Granato M., Moreau G., Wardenburg G, Bergman M. (2010), « Vocational Education and Trainig in Switzerland, France, Germany - Perspectives for Sociological, Rechearch ", Swiss Journal of Sociology, Volume 36/1, pp. 5-10.

Imdorf C. (2007), La sélection des apprentis dans les PME : Compte rendu mars 2007, Fribourg, Institut de Pédagogie Curative de l'université de Fribourg.

Lamamra N. (2016), Le genre de l'apprentissage, l'apprentissage du genre. Quand les arrêts prématurés révèlent les logiques à l'cuvre en formation professionnelle initiale, Zurich, Seismo.

Lamamra N. \& Masdonati J. (2009), Arrêter une formation professionnelle : mots et maux d'apprenti-e-s, Lausanne, Antipodes.

Levy R., Joye D., Guye O. \& Kaufmann V. (1997), Tous égaux ? De la stratification aux représentations, Zurich, Seismo.

Millet M. \& Moreau G. (2011), La société des diplômes, Paris, La Dispute.

Moreau G. (2003), Le monde apprenti, Paris, La Dispute.

Moser U. (2004), Jugentliche zwischen Schule und Berufsbildung: Eine Evaluation bei Schweizer Grossunternehmen unter Berücksichtigung des internationalen Schulleistungsvergleichs PISA. Leitungsgruppe des NFP 43. Retrieved June 01, 2005, from http://www.nfp43.unibe.ch

Müller B. \& Schweri J. (2012), Die Betriebe in der Berufsbildung: Entwicklungen 1985 bis 2008, Neuchâtel, Office fédéral de la statistique.

Office fédéral de la statistique (2015), Communiqué de presse. Travail et rémunération. Enquête suisse sur la population active et statistiques dérivées au troisième trimestre 2015 : l'offre de travail, Neuchâtel, OFS.

Palheta U. (2012), La domination scolaire. Sociologie de l'enseignement professionnel et de son public, Paris, Presses universitaires de France.

Strupler M. \& Wolter S. C.(2012), Die duale Lehre: eine Erfolgsgeschichte - auch für Betriebe. Ergebnisse der dritten Kosten-Nutzen-Erhebung der Lehrlingsausbildung aus Sicht der Betriebe, Chur/Zürich, Rüegger. 